Rochester Institute of Technology

RIT Scholar Works

Articles

Faculty \& Staff Scholarship

$1-22-2020$

\title{
Long Term Predictions of Coal Fired Power Plant Data Using Evolved Recurrent Neural Networks
}

\author{
Travis Desell \\ Rochester Institute of Technology \\ AbdEIRahman EISaid \\ Rochester Institute of Technology \\ Zimeng Lyu \\ Rochester Institute of Technology \\ David Stadem \\ Microbeam Technologies Inc. \\ Shuchita Patwardhan \\ Microbeam Technologies Inc.
}

See next page for additional authors

Follow this and additional works at: https://scholarworks.rit.edu/article

\section{Recommended Citation}

Desell, Travis J., ElSaid, AbdEIRahman A., Lyu, Zimeng, Stadem, David, Patwardhan, Shuchita and Benson, Steve. "Long term predictions of coal fired power plant data using evolved recurrent neural networks" at Automatisierungstechnik, vol. 68, no. 2, 2020, pp. 130-139. https://doi.org/10.1515/auto-2019-0116

This Article is brought to you for free and open access by the Faculty \& Staff Scholarship at RIT Scholar Works. It has been accepted for inclusion in Articles by an authorized administrator of RIT Scholar Works. For more information, please contact ritscholarworks@rit.edu. 


\section{Authors}

Travis Desell, AbdEIRahman EISaid, Zimeng Lyu, David Stadem, Shuchita Patwardhan, and Steve Benson 
Travis J. Desell*, AbdEIRahman A. ElSaid, Zimeng Lyu, David Stadem, Shuchita Patwardhan, and Steve Benson

\title{
Long Term Predictions of Coal Fired Power Plant Data Using Evolved Recurrent Neural Networks
}

\begin{abstract}
This work presents an investigation into the ability of recurrent neural networks (RNNs) to provide long term predictions of time series data generated by coal fired power plants. While there are numerous studies which have used artificial neural networks (ANNs) to predict coal plant parameters, to the authors' knowledge these have almost entirely been restricted to predicting values at the next time step, and not farther into the future. Using a novel neuro-evolution strategy called Evolutionary eXploration of Augmenting Memory Models (EXAMM), we evolved RNNs with advanced memory cells to predict per-minute plant parameters and per-hour boiler parameters up to 8 hours into the future. These data sets were challenging prediction tasks as they involve spiking behavior in the parameters being predicted. While the evolved RNNs were able to successfully predict the spikes in the hourly data they did not perform very well in accurately predicting their severity. The per-minute data proved even more challenging as medium range predictions miscalculated the beginning and ending of spikes, and longer range predictions reverted to long term trends and ignored the spikes entirely. We hope this initial study will motivate further study into this highly challenging prediction problem. The use of fuel properties data generated by a new Coal Tracker Optimization (CTO) program was also investigated and this work shows that their use improved predictive ability of the evolved RNNs.
\end{abstract}

Keywords: Neuro-Evolution, Recurrent Neural Networks, Time Series Data Prediction, Fuel Properties, Power Systems

*Corresponding author: Travis J. Desell, AbdEIRahman A. EISaid, Zimeng Lyu, Golisano College of Computing and Information Sciences, Rochester Institute of Technology, Rochester, NY, USA 14623,

David Stadem, Shuchita Patwardhan, Steve Benson, Microbeam Technologies Inc., Grand Forks, ND, USA 58203 


\section{Introduction}

Being able to predict operational parameters of coal fired power plants is an area of significant interest as accurate estimates can be used to improve plant efficient, reduce emissions or inform plant operators about conditions within the system. In particular, when conditions in the burners or other systems become poor due to coal quality or other effects, the operator may need to provide supplementary fuel to prevent the burner from going into shutdown. If these events can be predicted early enough then plant conditions can be modified to avoid shutdown without the use of supplementary fuel or other, potentially quite expensive, reactive methods.

Artificial neural networks (ANNs) have seen significant use in predicting plant parameters such as coal ash fusion temperatures [1], boiler parameters [2], corrosion rates [3], nitrogen oxide $\left(\mathrm{NO}_{\mathrm{x}}\right)$ emissions $[4,5,6,7,8,9]$, carbon monoxide (CO) [9], and air coefficients [10]. Apart from the work by Safdarnejad et al. [9] which utilizes a self generative model to predict $\mathrm{CO}$ and $\mathrm{NO}_{\mathrm{x}}$ up to three hours in the future, this work has been limited to predicting only the next time step into the future. Indeed, even some standard architectures such as Nonlinear Box-Jenkins (NBJ) [11] cannot even perform far future predictions as they require the error of previous predictions to be fed back into the network, which will not be known until that future time arrives.

This work investigates the long term predictive capabilities of recurrent neural networks (RNNs) by utilizing a novel neuro-evolution algorithm called EXAMM (Evolutionary eXploration of Augmenting Memory Models), which uses a suite of advanced recurrent memory cells, allowing for automated design of powerful RNN architectures which are optimized for their target prediction tasks. This work also examines the use of data generated by a new Coal Tracker Optimization (CTO) program which predicts the properties of coal as it is fed into the plant's burners. The plant's (net plant heat rate and nose gas temperature) were predicted using hourly data, and main flame intensity was predicted from per-minute data from a number of the plant's burners.

\section{Methodology}

Neuro-evolution, or the use of artificial evolutionary processes (such as genetic algorithms) to automate the design of ANNs, has been well applied to feed forward and convolutional neural networks $[12,13,14,15,16,17]$. However, less effort has been put into exploring the evolution of RNNs. 
Several neuro-evolution methods have been proposed for evolving RNN topologies (along with the weight values themselves) with NeuroEvolution of Augmenting Topologies (NEAT) [16] perhaps being the most well-known. Recent work by Rawal and Miikkulainen investigated an information maximization objective [18] strategy for evolving RNNs, which essentially operates similarly to NEAT except with LSTM cells being used instead of simple (traditional) neurons. Research centered around this line of NEAT-based approaches has also explored the use of a tree-based encoding [19] to evolve recurrent cellular structures within fixed architectures. More recently, work by Camero et al. has shown that a Mean Absolute Error (MAE) random sampling strategy can provide good estimates of RNN performance [20], successfully incorporating it into an LSTM-RNN neuro-evolution strategy [21]. Ant colony optimization (ACO) has also been used as a strategy to optimize RNNs by selecting subnetworks from within a larger fixed structure [22, 23].

For this study, EXAMM was selected as the RNN evolution algorithm for a number of reasons. In contrast to the well-known NEAT, EXAMM uses higher order node-level mutation operations, Lamarckian weight initialization (or the re-use of parental weights), and back-propagation through time (BPTT) to conduct local search, the combination of which has been shown to speed up both ANN training as well as the overall evolutionary process. Unlike the work by Rawal and Miikkulainen, EXAMM operates with an easily-extensible suite of memory cells, including LSTM, GRU, MGU, UGRNN, $\Delta$-RNN cells and, more importantly, has the natural ability to evolve deep recurrent connections over large, variable time lags which has recently been shown to provide significant improvements in predictive ability of RNNs [24]. In prior work it has also been shown to more quickly and reliably evolve RNNs in parallel than training traditional layered RNNs sequentially [25] and to significantly outperforming NEAT on time series prediction tasks. For detailed EXAMM implementation details we refer the reader to [26].

\section{Coal-fired Power Plant Data}

This work uses data from both the boiler and burners of a coal fired power plant, which was collected by Microbeam Technologies, Inc. (MTI).

\subsection{Burner Data Set}

The main burner data set consists of 10 days of data extracted from 12 of the plant's burners. Each of these 12 data files consisted of time series data from 12 sensors, 
recorded each minute (see Appendix A). To expand on these 12 sensor parameters, Microbeam developed a program named Coal Tracker Optimization (CTO) which simulates and projects as-fired coal quality at the burner [27, 28]. Coal quality was collected from an online full stream elemental analyzer (FSEA) positioned at the entry point to the fuel handling system at the plant. The program uses live data from the plant to calculate the residence time of coal as it flows from the FSEA through the coal delivery system and finally to the burner inlet. The CTO program was designed using a mechanistic model that implemented abstractions of different components in the coal tracking system. To calibrate the CTO program, coal samples were collected at four burners and the analysis results were compared to the calculated as-fired fuel quality from CTO. It was of particular interest to see if the additional fuel properties information would improve predictions. The additional time series variables provided by CTO are in Appendix B.

From this burner data set, main flame intensity was the parameter of interest for prediction. It is measured by optical pyrometers positioned near the entry point of coal into the burner. Changes in operational parameters often induce dramatic changes in flame intensity: for example, instantaneous drops in flame intensity are seen when the burner is turned off. Changing fuel properties can cause changes in flame intensity on different time-scales, ranging from a few minutes to a few hours. The impact of fuel properties on combustion is well-studied; however, it is rare for a plant to monitor day-by-day or minute-by-minute fluctuations in fuel properties at the burner. It is of particular interest in that sometimes planned outages occur or conditions in the burner deteriorate so badly that it is temporarily shut down. In these cases, sharp spikes occur during the shutdown, which last for an unspecified period of time before the burner turns back on again and the flame intensity value sharply increases.

\subsection{Boiler Data Set}

The boiler data set consisted of over a year of per-hour data readings from the plant's on-line coal analyzers, coal handling system, and steam generator. This data divided into five separate data files, each representing a different period of operation. These were 1688, 1289, 1522, 902 and 1997 hours long. This work focused on predicting two different parameters which can be used in calculating boiler efficiency, net plant heat rate and nose gas temperature. In total, the data set consisted of 53 different input parameters (see Appendix C). 


\section{Results}

EXAMM was compared to standard Nonlinear Autoregressive Exogenous (NARX) and Nonlinear Output Error (NOE) models which only achieved error rates of $18.65 \%$ and $18.75 \%$, respectively, for 1 hour nose gas temperature predictions, and $24.3 \%$ and $24.8 \%$ for 1 hour heat rate predictions. For 1 minute predictions of main flame intensity on the Burner data set, NARX and NOE only reached error rates of $15.1 \%$ and $14.3 \%$, respectively, for Cyclone 1 and $10.2 \%$ and $10.2 \%$ for Cyclone 3. As these results were up to an order of magnitude worse than EXAMM these models were not further pursued.

\subsection{Experiments}

All the experiments performed used the same EXAMM and backpropagation hyperparameters as described in prior work [26]. All EXAMM runs selected from all possible neuron types (simple neurons, $\triangle$-RNN, GRU, MGU, LSTM and UGRNN memory cells) uniformly at random, which allowed EXAMM to select the most effective architectural and memory components. Each run of EXAMM generated and trained 10,000 RNNs as part of the neuro-evolutionary process, and was repeated 10 times to better account for the stochastic nature of the search. All experiments were done using Rochester Institute of Technology's SPORK high performance computing cluster and were distributed across 20 compute nodes, each with 36 cores for a total of 720 cores used by each run.

\subsection{Boiler Parameter Predictions}

For evaluating the predictive of the evolved RNNs on the boiler data, the first four data files were used as training data and the last and longest (the 1997 hour data file) was used as test data. Separate experiments were done using EXAMM to predict the nose gas temperature and net plant heat rate parameters 1,2, 4 and 8 hours into the future. Figure 1 presents the range of the error rates of the best evolved RNNs on the test data after $2 \mathrm{k}, 5 \mathrm{k}$ and $10 \mathrm{k}$ trained RNNs. As expected, predicting farther into the future resulted in higher error rates. Net plant heat rate and significantly lower error, under $1 \%$ even at 8 hours, while nose gas temperature increased from $3 \%$ to $7 \%$ as prediction time increased from 1 to 8 hours.

Figure 2 presents the predictions of the best found RNNs evolved by EXAMM for each prediction offset. All these RNNs were fairly effective at capturing the sharp changes in net plant heat rate and nose gas temperature, however the farther 


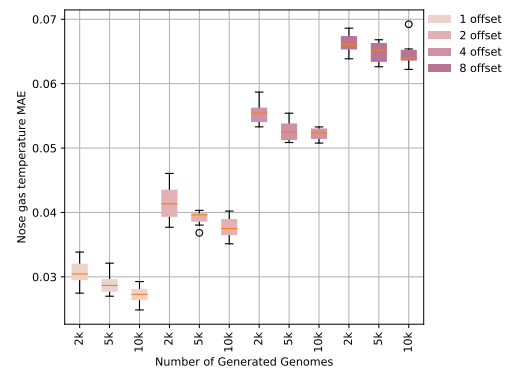

(a) Nose Gas Temperature

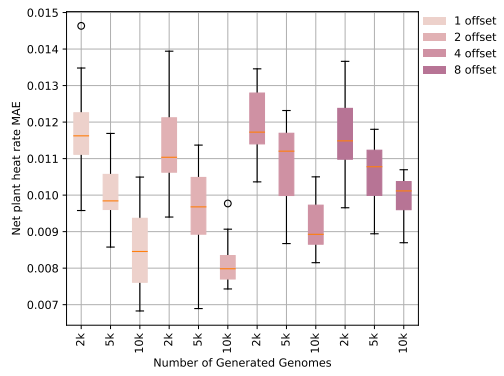

(b) Net Plant Heat Rate

Fig. 1: Mean absolute error (MAE) rates for the boiler parameters for a range of time offsets into the future (1 hour to 8 hours).

the prediction was into the future the less accurate it was in determining the beginning of a spike as well as calculating the minimum or maximum value the spike would reach. The prediction plots also show why the RNNs performed worse on nose gas temperature, as the spikes within this data were much more dramatic making for a highly challenging prediction problem.

\subsection{Flame Intensity Predictions}

Determining the effect of using the additional CTO generated fuel properties parameters to predict main flame intensity was of particular interest. Three separate types of runs were done utilizing various sets of input parameters. The first used only the burner parameters, the second used all the burner parameters and fuel properties parameters and the last used a reduced set of burner parameters along with the fuel properties parameters. These were labeled as Burner, Burner + Fuel and Burner $\sim$ Fuel, respectively. RNNs were trained on the first 7.5 days of the data sets, and validated on the remaining 2.5 days, allowing enough time for fuel and plant data to be retained by the RNNs to predict spiking events.

While EXAMM can remove input parameters which are unneeded or confounding during the neuro-evolution process, having almost twice as many input parameters between the data sets could provide for a larger search space and potentially slow how fast EXAMM finds well performing RNNs. Because of this, the additional Burner $\sim$ Fuel set of input parameters was tested, which removed out the tertiary air split, secondary air split, secondary air ratio, system secondary air flow total, primary air split, tertiary air flow, primary air flow and conditioner 


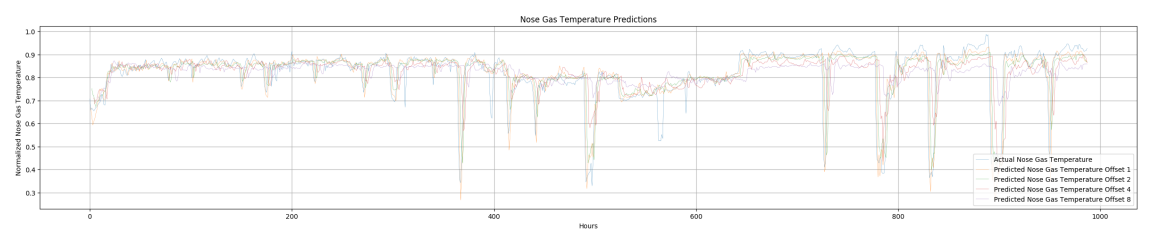

(a) Nose Gas Temperature

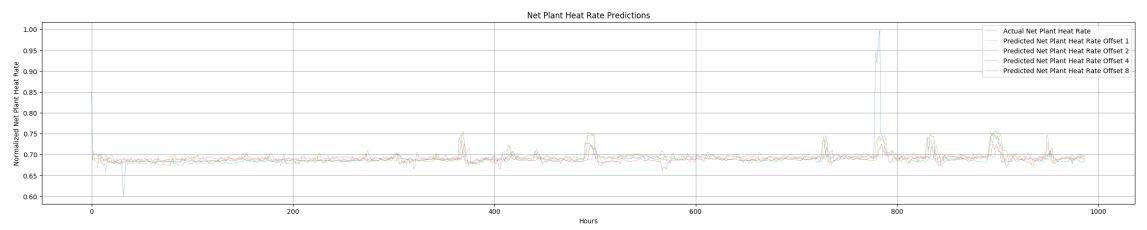

(b) Net Plant Heat Rate

Fig. 2: Predictions of the evolved RNNs on the boiler parameters, predicting 1 to 8 hours into the future.

outlet temp variables resulting in the same number of input parameters as the Burner runs, as these were shown to have the least statistical significance to main flame intensity in cross-correlation tests.

Figure 3 presents the mean absolute error (MAE) of the ten repeated runs predicting flame intensity $1,15,30,60,120,240$, and 480 minutes into the future for a representative selection of 2 cyclones (cyclones 1 and 3 ) for each of the three sets of possible input parameters. While these represent similar time ranges of prediction as done for the boiler parameters (up to 8 hours), they do present a much more complicated learning task for the RNNs due to the higher time frequency of recordings - the memory cells and recurrent connections need to remember prior important information for significantly more time steps (480 vs 8 , in the example of 8 hours). As examples, Figure 4 shows the predictions made by the best evolved RNNs for each input parameter set and prediction time offset.

Predictions were fairly accurate, ranging from $3.5 \%$ MAE in the best case for 1 minute predictions and up to $10 \% \mathrm{MAE}$ for longer term predictions. Across nearly every experiment, the Burner + Fuel input parameters outperformed the other two input parameter sets, which show that the additional data provided by the CTO program was improving predictions. Further, it shows that EXAMM is performing well with the increased parameters which did not seem to degrade its performance.

While many of the cyclones saw increasing error rates for longer term predictions, on some cyclones the error rates decreased for longer term predictions (as shown in cyclone 1), which was quite surprising. However, as can be seen in 


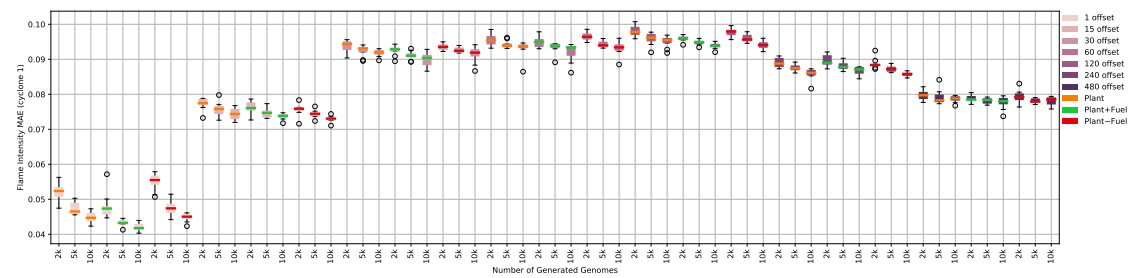

(a) Cyclone 1

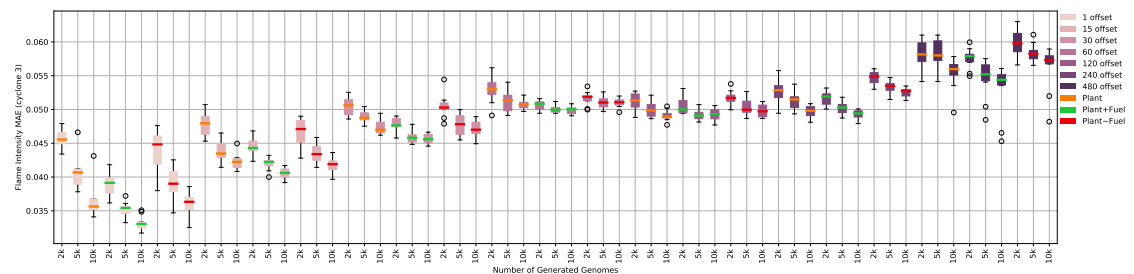

(b) Cyclone 3

Fig. 3: Mean absolute error (MAE) rates for a selection of cyclones with various input parameters for a range of time offsets into the future (1 minute to 480 minutes).

Figure 4, this is unfortunately due to the RNNs learning average trends and simply ignoring potential spikes. While the predictions 1 minute into the future follow spikes very well, those done 15 or 30 minutes into the future, especially for cyclone 1 lag behind the spikes and do not appear to be able to anticipate the upcoming spikes - which could be very important information to improve plant operations.

\section{Conclusions}

This work presents a study investigating the capabilities of recurrent neural networks for predicting time series data farther in the future than the next time step of data. Additionally, instead of simply training fixed traditional architectures, a powerful neuro-evolution algorithm called EXAMM was utilized to automate the design and selection of recurrent memory cell types to provide the best possible predictions. This work provides some positive results, in that the use of new fuel properties data generated by a new Coal Tracker Optimization (CTO) program improved the RNNs predictive abilities and that EXAMM was not hindered by having additional input parameters to select from. However, it does also illustrate challenges which will drive future work in this area. For the prediction of boiler parameters using 


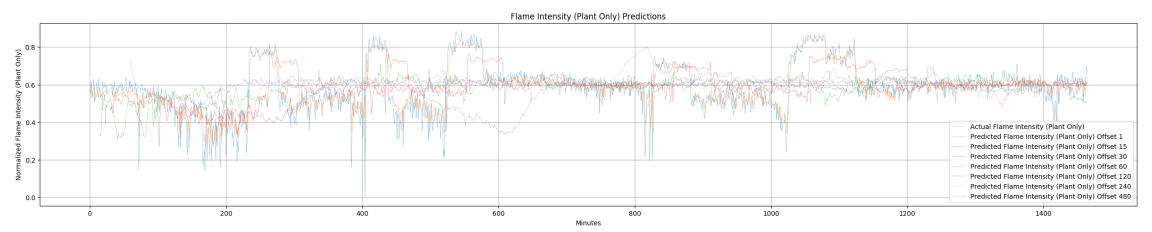

(a) Cyclone 1 - Burner Parameters Only

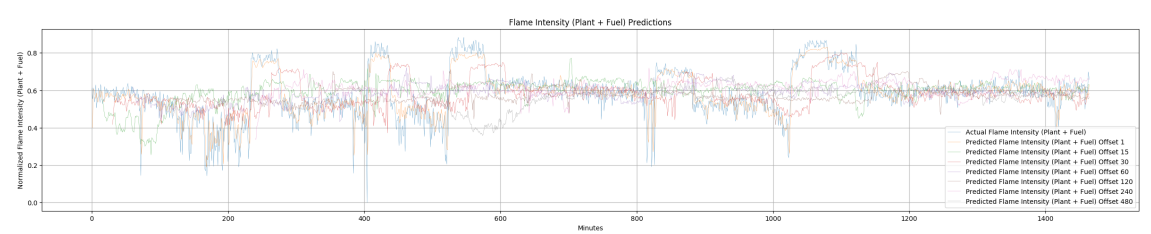

(b) Cyclone 1 - Burner Parameters + Fuel Properties

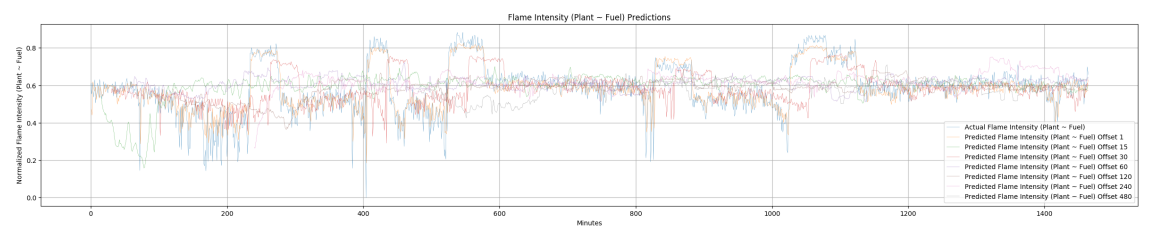

(c) Cyclone 1 - Burner Parameters $\sim$ Fuel Properties (Reduced)

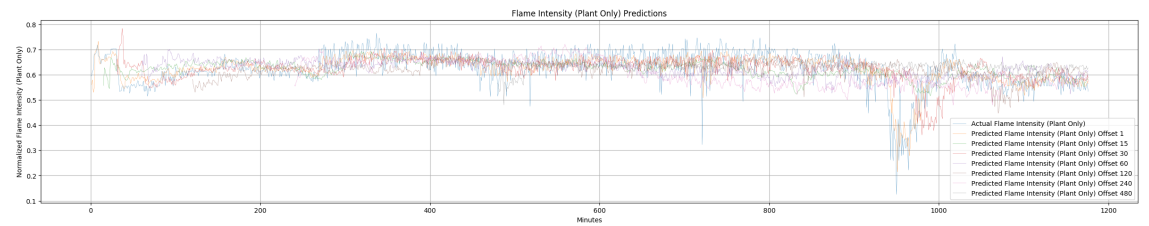

(d) Cyclone 3 - Burner Parameters Only

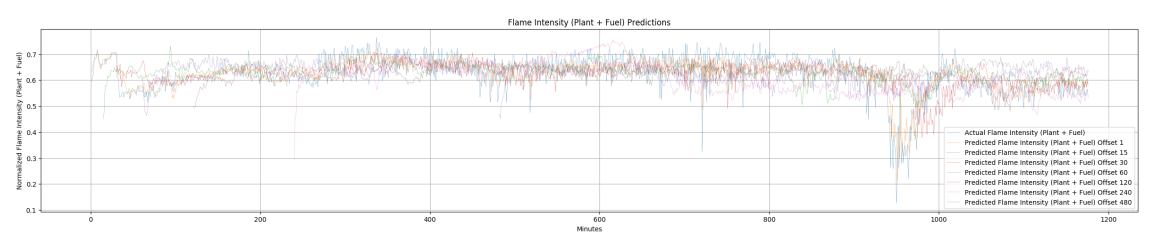

(e) Cyclone 3 - Burner Parameters + Fuel Properties

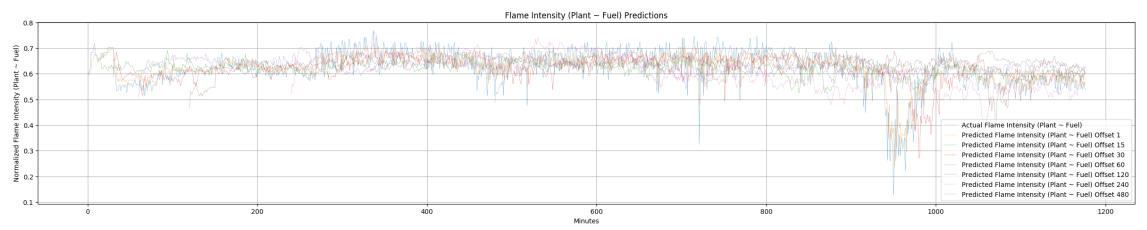

(f) Cyclone 3 - Burner Parameters $\sim$ Fuel Properties (Reduced)

Fig. 4: Predictions of the evolved RNNs on the boiler parameters for cyclone 3, predicting 1 to 8 hours into the future 
hourly data, it was shown that the RNNs were capable of predicting most spikes in the data, however the longer term predictions performed more poorly in accurately capturing the magnitude of those spikes. Predicting burner data with a per-minute frequency proved to be an even more challenging task. RNNs were very capable of predicting one minute into the future, however in the mid range (15 minutes to 1 hour), RNNs lagged behind capturing spiking events and did not anticipate them. For the longer term predictions ( 2 to 8 hours), the RNNs simply ignored the spiking events and instead predicted average trends. Given these difficulties, improving these longer term predictions makes for an interesting and challenging avenue of future work. A major area of potential benefit is to use future coal property forecasts from CTO and combine them with current time values of burner parameters, as this study only used fuel properties up to the time the prediction was made. Other potential areas to study will include varying the frequencies of the input data, e.g., using hourly averages instead of per minute data, as well as enhancing EXAMM with more powerful mutation operations such as adding entire layers of memory cells at once and co-evolving memory cell structures instead of using fixed traditional memory cells.

Acknowledgment: This material is in part supported by the U.S. Department of Energy, Office of Science, Office of Advanced Combustion Systems under Award Number \#FE0031547. We also thank Microbeam Technologies, Inc., for their help in collecting and preparing the coal-fired power plant.

\section{References}

[1] Michalis Mavrovouniotis and Shengxiang Yang. Evolving neural networks using ant colony optimization with pheromone trail limits. In Computational Intelligence (UKCI), 2013 13th UK Workshop on, pages 16-23. IEEE, 2013.

[2] J Smrekar, D Pandit, Magnus Fast, Mohsen Assadi, and Sudipta De. Prediction of power output of a coal-fired power plant by artificial neural network. Neural Computing and Applications, 19(5):725-740, 2010.

[3] Amrita Kumari, SK Das, and PK Srivastava. Modeling fireside corrosion rate in a coal fired boiler using adaptive neural network formalism. Portugaliae Electrochimica Acta, 34(1):23-38, 2016.

[4] Hao Zhou, Kefa Cen, and Jianren Fan. Modeling and optimization of the NOx emission characteristics of a tangentially fired boiler with artificial neural networks. Energy, 29(1):167 - 183, 2004.

[5] Fang Wang, Suxia Ma, He Wang, Yaodong Li, and Junjie Zhang. Prediction 
of NOx emission for coal-fired boilers based on deep belief network. Control Engineering Practice, 80:26-35, 2018.

[6] Zhou Hao, Cen Kefa, and Mao Jianbo. Combining neural network and genetic algorithms to optimize low no x pulverized coal combustion. Fuel, 80(15):21632169, 2001.

[7] Jiyu Chen, Feng Hong, Mingming Gao, Taihua Chang, and Liying Xu. Prediction model of scr outlet NOx based on LSTM algorithm. In Proceedings of the 2019 2nd International Conference on Intelligent Science and Technology, pages 7-10. ACM, 2019.

[8] Peng Tan, Biao He, Cheng Zhang, Debei Rao, Shengnan Li, Qingyan Fang, and Gang Chen. Dynamic modeling of NOx emission in a $660 \mathrm{MW}$ coal-fired boiler with long short-term memory. Energy, 176:429-436, 2019.

[9] Seyed Mostafa Safdarnejad, Jake F Tuttle, and Kody M Powell. Dynamic modeling and optimization of a coal-fired utility boiler to forecast and minimize NOx and CO emissions simultaneously. Computers \& Chemical Engineering, 124:62-79, 2019.

[10] Cem Onat and Mahmut Daskin. A basic ann system for prediction of excess air coefficient on coal burners equipped with a ccd camera. Mathematics and Statistics, 7(1):1-9, 2019.

[11] Peter J Brockwell, Richard A Davis, and Stephen E Fienberg. Time Series: Theory and Methods: Theory and Methods. Springer Science \& Business Media, 1991.

[12] Khalid Salama and Ashraf M Abdelbar. A novel ant colony algorithm for building neural network topologies. In Swarm Intelligence, pages 1-12. Springer, 2014.

[13] Masanori Suganuma, Shinichi Shirakawa, and Tomoharu Nagao. A genetic programming approach to designing convolutional neural network architectures. In Proceedings of the Genetic and Evolutionary Computation Conference, GECCO '17, pages 497-504, New York, NY, USA, 2017. ACM.

[14] Yanan Sun, Bing Xue, and Mengjie Zhang. Evolving deep convolutional neural networks for image classification. CoRR, abs/1710.10741, 2017.

[15] Risto Miikkulainen, Jason Liang, Elliot Meyerson, Aditya Rawal, Dan Fink, Olivier Francon, Bala Raju, Hormoz Shahrzad, Arshak Navruzyan, Nigel Duffy, and Babak Hodjat. Evolving deep neural networks. arXiv preprint arXiv:1703.00548, 2017.

[16] Kenneth Stanley and Risto Miikkulainen. Evolving neural networks through augmenting topologies. Evolutionary computation, 10(2):99-127, 2002.

[17] Kenneth O Stanley, David B D'Ambrosio, and Jason Gauci. A hypercube-based encoding for evolving large-scale neural networks. Artificial life, 15(2):185-212, 2009 . 
[18] Aditya Rawal and Risto Miikkulainen. Evolving deep LSTM-based memory networks using an information maximization objective. In Proceedings of the Genetic and Evolutionary Computation Conference 2016, pages 501-508. ACM, 2016.

[19] Aditya Rawal and Risto Miikkulainen. From nodes to networks: Evolving recurrent neural networks. CoRR, abs/1803.04439, 2018.

[20] Andrés Camero, Jamal Toutouh, and Enrique Alba. Low-cost recurrent neural network expected performance evaluation. arXiv preprint arXiv:1805.07159, 2018.

[21] Andrés Camero, Jamal Toutouh, and Enrique Alba. A specialized evolutionary strategy using mean absolute error random sampling to design recurrent neural networks. arXiv preprint arXiv:1909.02425, 2019.

[22] Travis Desell, Sophine Clachar, James Higgins, and Brandon Wild. Evolving deep recurrent neural networks using ant colony optimization. In European Conference on Evolutionary Computation in Combinatorial Optimization, pages 86-98. Springer, 2015.

[23] AbdElRahman ElSaid, Fatima El Jamiy, James Higgins, Brandon Wild, and Travis Desell. Optimizing long short-term memory recurrent neural networks using ant colony optimization to predict turbine engine vibration. Applied Soft Computing, 2018.

[24] Travis J. Desell, AbdElRahman A. ElSaid, and Alexander G. Ororbia. An empirical exploration of deep recurrent connections and memory cells using neuro-evolution, 2019.

[25] AbdElRahman ElSaid, Steven Benson, Shuchita Patwardhan, David Stadem, and Desell Travis. Evolving recurrent neural networks for time series data prediction of coal plant parameters. In The 22nd International Conference on the Applications of Evolutionary Computation, Leipzig, Germany, April 2019.

[26] Alexander Ororbia, AbdElRahman ElSaid, and Travis Desell. Investigating recurrent neural network memory structures using neuro-evolution. In Proceedings of the Genetic and Evolutionary Computation Conference, GECCO '19, pages 446-455, New York, NY, USA, 2019. ACM.

[27] Shuchita Patwardhan, David Stadem, Matt Fuka, and Steve Benson. Condition Based Monitoring and Predicting Ash Behavior in Coal Fired Boilers -II Coal Properties Optimization. Paper Presented at Clearwater Clean Energy Conference, June 2019.

[28] David Stadem, Shuchita Patwardhan, Matt Fuka, James Langfeld, Alek Benson, and Steven Benson. Improving Coal Fired Plant Performance Using a Coal Tracker Optimization Tool. Paper Presented at Pittsburgh Coal Conference, September 2019. 


\section{Appendix}

\section{A Burner Parameters}
1. Conditioner Inlet Temp
7. Secondary Air Flow
2. Conditioner Outlet Temp
8. Secondary Air Split
3. Coal Feeder Rate
9. Tertiary Air Split
4. Primary Air Flow
10. Total Combined Air Flow
5. Primary Air Split
11. Supplementary Fuel Flow
6. System Secondary Air Flow Total
12. Main Flame Intensity

\section{B Fuel Properties Parameters}
1. Base Acid Ratio
2. Ash Content
3. Na (Sodium) Content
4. Fe (Iron) Content

\section{Boiler Parameters}

1. Cold Reheat Steam Temperature

2. ECON Flue Gas Out Press

3. ECON HDR 01 Out Temp

4. ECON HDR 02 Out Temp

5. ECON In Gas Temp

6. Economizer Differential

7. Economizer Gas Outlet O2 Level

8. Economizer Inlet Feedwater Flow

9. Economizer Inlet Feedwater Temperature

10. Economizer Outlet Avg

11. Economizer Gas Recirc Outlet Temperature
5. BTU
6. Ash Flow
7. Na (Sodium) Flow
8. Fe (Iron) Flow

12. Fuel Cost For $1 \mathrm{Btu} / \mathrm{Kwh}$ Heat Rate Deviation

13. Gross Generator Output

14. Hot Reheat Temperature (Reheater Outlet)

15. Main Steam Pressure At Boiler

16. Main Steam Temp (Superheater Outlet)

17. Main Steam Press

18. Main Steam Spray Flow

19. Main Steam Spray Press

20. NOx Master Out

21. Net Plant Heat Rate

22. Net Unit Generation 
23. Nose Gas Temperature

24. Prim Suphtr Differential

25. PSH Gas Outlet Temperature

26. PSH Outlet Avg

27. PSH Superheater Gas Inlet Temperature

28. RH Suphtr Bank-1 Diff

29. RH Suphtr Bank-2 Diff

30. Sec SH Inlet Temp Avg

31. Sec SH Outlet Temp Avg

32. SSH Inlet HDR 01 Temp

33. SSH Inlet HDR 04 Temp

34. SSH Out HDR TC 01 Temp

35. SSH Out HDR TC 02 Temp

36. SSH Out HDR TC 03 Temp

37. SSH Out HDR TC 04 Temp
38. SSH Outlet HDR TC 05 Temp

39. Total OFA Air Flow

40. Water Wall Raw Cleanliness

41. Avg Conditioner Inlet Temp

42. Avg Conditioner Outlet Temp

43. Total Lignite Feeder Rate

44. Total Primary Air Flow

45. Avg Primary Air Split

46. Total Secondary Air Flow

47. Avg Secondary Air Split

48. Total Tertiary Air Flow

49. Avg Tertiary Air Split

50. Total Combined Air Flow

51. Total Main Oil Flow

52. Avg Main Flame Intensity

53. Time Until Next Shutdown 